\section{Condutas de saúde entre universitários: \\ diferenças entre gêneros}

\author{
Health-related behavior in a sample of Brazilian \\ college students: gender differences
}

\footnotetext{
1 Universidade de Pernambuco, Recife, Brasil.

Correspondência V. Colares

Universidade de Pernambuco. Rua Barão de Itamaracá 460, apto. 401, Recife, $P E$ 52020-070, Brasil. vcolares@elogica.com.br
}

\begin{abstract}
This study investigated whether undergraduate students' health-risk behaviors differed according to gender. The sample consisted of $382 \mathrm{sub}$ jects, aged 20-29 years, from public universities in Pernambuco State, Brazil. Data were collected using the National College Health Risk Behavior Survey, previously validated in Portuguese. Descriptive and inferential statistical techniques were used. Associations were analyzed with the chi-square test or Fisher's exact test. Statistical significance was set at $p \leq 0.05$. In general, females engaged in the following risk behaviors less frequently than males: alcohol consumption ( $p=0.005)$, smoking $(p=0.002)$, experimenting with marijuana ( $p=0.002)$, consumption of inhalants ( $p \leq 0.001)$, steroid use ( $p=0.003)$, carrying weapons $(p=0.001)$, and involvement in physical fights $(p=0.014)$. Meanwhile, female students displayed more concern about losing or maintaining weight, although they exercised less frequently than males. The findings thus showed statistically different health behaviors between genders. In conclusion, different approaches need to be used for the two genders.
\end{abstract}

Health Occupations; Students; Health Behavior; Risk Factors; Gender
Viviane Colares 1

Carolina da Franca 1

Emília Gonzalez 1

\section{Introdução}

As condutas de saúde repercutem de forma significativa na qualidade de vida dos indivíduos, especialmente por sua relação com as doenças crônicas. Observam-se diferenças entre os gêneros nos comportamentos relacionados à saúde, enquanto os homens relatam praticar mais freqüentemente exercícios físicos, as mulheres demonstram significativamente maior preocupação com a dieta alimentar 1 .

Segundo Rugkasa et al. 2, as identidades dos gêneros masculino e feminino se desenvolvem ao longo da vida do indivíduo quando diferentes caminhos podem ser adotados com repercussões nas condutas relacionadas aos hábitos, como por exemplo, tabagismo.

Alguns estudos investigaram as condutas de saúde entre jovens universitários e verificaram diferenças entre gêneros, destacando a necessidade de estratégias diferenciadas para cada grupo $3,4,5$.

Os estudantes do gênero masculino demonstraram em alguns estudos a adoção de condutas menos saudáveis como, o tabagismo 6,7 , o consumo de álcool 4,8 e o abuso de substâncias 4 . Com relação ao peso corporal, apesar do sobrepeso ter sido mais prevalente entre os homens, as mulheres se auto-avaliaram mais freqüentemente como acima do peso, embora tenham apresentado um índice de massa corporal (IMC) normal 9 . 
Silva et. al. 10 investigaram fatores associados ao consumo de drogas lícitas e ilícitas entre universitários, em uma população de 924 estudantes de ciências biológicas na cidade de São Paulo. Observou-se alto consumo de bebida alcoólica $(84,7 \%)$ e de tabaco $(22,8 \%)$, sem diferenças significativas entre os gêneros. No entanto, entre as drogas ilícitas, os homens apresentaram significativamente $(\mathrm{p}<0,001)$ maior consumo $(36,8 \%)$ do que as mulheres (23\%).

Por outro lado, as mulheres também demonstraram algumas condutas pouco saudáveis como: baixa atividade física 11 e atividade sexual desprotegida 12 .

Este estudo teve como objetivo investigar diferenças entre os gêneros nas condutas de saúde entre universitários da área de saúde.

\section{Métodos}

A amostra foi composta por universitários na faixa etária de 20 a 29 anos, estudantes do final de cursos da área de saúde das universidades públicas do Estado de Pernambuco, Brasil. Foram considerados estudantes do final do curso aqueles matriculados no último semestre com disciplinas teóricas. Tal critério foi adotado para que todos os alunos pudessem responder ao questionário em sala de aula.

O cálculo amostral foi estratificado por curso e por gênero, considerando: o objetivo de determinação dos percentuais (prevalências) dos fatores de risco; prevalências obtidas na amostra da pesquisa mais próximas de $50 \%$; margem de erro de $5 \%$; confiabilidade de que a margem de erro não seja ultrapassada; o número de alunos em cada gênero nas duas universidades. Amostra calculada totalizou 224 universitários, sendo 79 (35,3\%) do gênero masculino e 145 (64,7\%) do gênero feminino.

A coleta de dados foi realizada entre os meses de abril e agosto de 2006 mediante a aplicação do questionário de forma individual, anônima e autopreenchível em sala de aula, na disciplina que apresentou o maior número de alunos matriculados.

O instrumento de coleta de dados foi o questionário National College Health Risk Behavior Survey (NCHRBS), desenvolvido pelo Centers for Disease Control and Prevention (CDC) dos Estados Unidos, previamente validado para utilização com 208 jovens universitários brasileiros. O processo de validação foi realizado em cinco etapas: (1) tradução; (2) retrotradução; (3) correção e adaptação semântica (transculturação); (4) validação de face; e (5) teste e reteste.
O kappa médio para todos os tópicos foi 0,76 , classificado como bom.

Este questionário é composto por informações sócio-demográficas e pelos seguintes temas relacionados à saúde: segurança no trânsito, comportamentos relacionados à violência, consumo de drogas lícitas (álcool e tabaco) e ilícitas, comportamento sexual, hábitos alimentares, percepção do peso e prática de atividade física.

O tabagismo regular foi considerado consumo de no mínimo um cigarro por semana pelo período de um mês. O hábito alimentar foi baseado na dieta do dia anterior, assim como a prática de atividade física foi avaliada segundo o histórico da semana anterior à coleta de dados. Esses parâmetros foram os mesmos adotados pelo CDC 13 .

Para a análise dos dados foram utilizadas técnicas de estatística descritiva e inferencial. Inicialmente, foi realizada uma análise exploratória univariada para observar a distribuição de freqüências das variáveis estudadas. Para a análise de associação entre variáveis foi utilizado o teste qui-quadrado ou o teste exato de Fisher, quando as condições para a utilização do teste qui-quadrado não foram verificadas. Os resultados foram considerados estatisticamente significantes para um valor de $\mathrm{p}<0,05$.

Os dados foram digitados na planilha Excel (Microsoft Corp., Estados Unidos), e o programa utilizado para a obtenção dos cálculos estatísticos foi o Statistical Package for the Social Science (SPSS) na versão 13.0 (SPSS Inc., Chicago, Estados Unidos).

O termo de consentimento livre e esclarecido foi assinado pelos participantes voluntariamente, sendo garantido anonimato e sigilo. O projeto de pesquisa foi aprovado pelo Comitê de Ética da Universidade de Pernambuco (protocolo $n^{\circ}$. 087/04) e esteve de acordo com os princípios éticos contidos na Declaração de Helsinki.

\section{Resultados}

No processo de coleta de dados, obteve-se uma amostra maior do que a calculada inicialmente, dessa forma participaram do estudo 382 estudantes dos nove cursos de saúde da Universidade Federal de Pernambuco - UFPE (Fisioterapia, Nutrição, Odontologia, Medicina, Enfermagem, Educação Física, Fonoaudiologia, Farmácia e Terapia Ocupacional) e dos quatro cursos da Universidade de Pernambuco - UPE (Odontologia, Medicina, Enfermagem e Educação Física).

A idade dos 266 pesquisados do sexo feminino variou de 20 a 28 anos, teve média de 23,30 anos, desvio padrão de 1,58 ano, coeficiente de 
variação de 6,78 e a idade mediana foi 23,00 anos. Para os 116 pesquisados do sexo masculino a idade variou de 21 a 29 anos, com média de 23,96 anos e desvio padrão de 1,82 ano, coeficiente de variação de 7,58 e a idade mediana foi 24,00 anos.

A escolaridade da mãe foi significativamente menor entre estudantes do gênero feminino ( $\mathrm{p}=$ 0,002), enquanto a escolaridade do pai não apresentou diferença significante entre os gêneros $(\mathrm{p}=0,156)$.

As condutas relacionadas à segurança no trânsito evidenciaram atitudes de risco, como andar em veículo cujo motorista consumiu bebida alcoólica ou dirigir um carro após consumir bebida alcoólica, não apresentando diferença significativa entre os gêneros. Com relação à violência, as condutas relatadas apresentaram baixos percentuais, sendo um pouco mais elevadas entre estudantes do gênero masculino (Tabela 1).

O álcool e o tabaco foram consumidos pela maioria dos estudantes, sendo os percentuais significativamente mais elevados entre os estudantes do gênero masculino (Tabela 2).
Quanto às condutas relacionadas ao uso de drogas ilícitas, verificou-se que o uso de maconha foi menos freqüente entre estudantes do gênero feminino, assim como o uso de inalantes e de injeções de esteróides (Tabela 3 ).

A prática de atividade sexual na vida foi significativamente mais freqüente entre estudantes do gênero masculino, assim como o consumo de álcool ou drogas antes da última relação sexual ( $p=0,005)$ (Tabela 4$)$. No entanto, em relação à prática de atividade sexual nos trinta dias anteriores à pesquisa, não houve diferença significante entre os gêneros $(\mathrm{p}=0,301)$.

Com relação à auto-avaliação do peso, as mulheres relataram mais freqüentemente que estavam tentando perder peso, enquanto os homens estavam tentando ganhar (Tabela 5).

Em relação à alimentação, não houve diferença significante entre os gêneros; a prática de atividade física, porém, foi significativamente mais freqüente entre os estudantes do gênero masculino (Tabela 5).

Avaliação das condutas relacionadas à segurança no trânsito e à violência por gênero.

\begin{tabular}{|c|c|c|c|c|c|}
\hline \multirow[t]{3}{*}{ Condutas } & \multicolumn{4}{|c|}{ Gênero } & \multirow[t]{3}{*}{ Valor de $p$} \\
\hline & \multicolumn{2}{|c|}{ Masculino } & \multicolumn{2}{|c|}{ Feminino } & \\
\hline & n (116) & $\%$ & n (266) & $\%$ & \\
\hline \multicolumn{6}{|l|}{ Uso do cinto de segurança no banco da frente do carro } \\
\hline Nunca & - & - & - & - & 0,304 * \\
\hline Raramente/Às vezes & 1 & 0,9 & - & - & \\
\hline A maioria das vezes/Sempre & 115 & 99,1 & 266 & 100,0 & \\
\hline \multicolumn{6}{|l|}{ Uso do cinto de segurança no banco traseiro do carro } \\
\hline Nunca & 15 & 12,9 & 24 & 9,0 & 0,480 ** \\
\hline Raramente/Às vezes & 81 & 69,8 & 190 & 71,4 & \\
\hline A maioria das vezes/Sempre & 20 & 17,2 & 52 & 19,5 & \\
\hline \multicolumn{6}{|l|}{ Uso do capacete ao andar de bicicleta no último ano *** } \\
\hline Nunca & 60 & 88,2 & 85 & 90,4 & 0,217 * \\
\hline Raramente/Às vezes & 3 & 4,4 & 7 & 7,4 & \\
\hline A maioria das vezes/Sempre & 5 & 7,4 & 2 & 2,1 & \\
\hline $\begin{array}{l}\text { Andar em um veículo dirigido por você ou outra pessoa após consumir } \\
\text { bebida alcoólica \# }\end{array}$ & 75 & 65,2 & 173 & 65,0 & $0,973 * \star$ \\
\hline Carregar uma arma de fogo/faca nos últimos 30 dias & 7 & 6,0 & 1 & 0,4 & 0,001 * \\
\hline Envolver-se em uma briga física nos últimos 12 meses & 11 & 9,5 & 9 & 3,4 & 0,014 * \\
\hline Tentativa de suicídio no último ano & - & - & 4 & 1,5 & 0,319 * \\
\hline
\end{tabular}

* Emprego do teste exato de Fisher;

** Emprego do teste qui-quadrado de Pearson;

*** 220 pesquisados não andaram de bicicleta nos últimos 12 meses (48 masculinos, 172 femininos);

\# 1 pesquisado não respondeu à questão (masculino). 
Avaliação do consumo de tabaco e álcool segundo o gênero.

\begin{tabular}{|c|c|c|c|c|c|}
\hline \multirow[t]{3}{*}{ Condutas } & \multicolumn{4}{|c|}{ Gênero } & \multirow[t]{3}{*}{ Valor de $\mathrm{p}$} \\
\hline & \multicolumn{2}{|c|}{ Masculino } & \multicolumn{2}{|c|}{ Feminino } & \\
\hline & n (116) & $\%$ & n (266) & $\%$ & \\
\hline Consumo na vida do tabaco & 71 & 61,2 & 130 & 48,9 & $0,026 *, * *$ \\
\hline Consumo freqüente do tabaco *** & 23 & 32,4 & 18 & 13,8 & $0,002 *, * \star$ \\
\hline \multicolumn{6}{|l|}{ 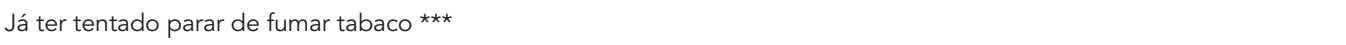 } \\
\hline Nunca fumou regularmente & 50 & 70,4 & 118 & 90,8 & $0,001 *, \star *$ \\
\hline $\operatorname{Sim}$ & 15 & 21,1 & 7 & 5,4 & \\
\hline Não & 6 & 8,5 & 5 & 3,8 & \\
\hline Consumo na vida do álcool & 106 & 91,4 & 212 & 79,7 & $0,005 *, \star \star *$ \\
\hline Consumo de bebida alcoólica nos últimos 30 dias\# & $\$ 89$ & 84,0 & 164 & 77,7 & 0,192 ** \\
\hline
\end{tabular}

* Associação significante a 5\%;

** Emprego do teste qui-quadrado de Pearson;

*** Apenas os participantes que já fumaram responderam à questão;

\# 65 participantes não responderam à questão (10 masculinos e 55 femininos)

Consumo de drogas ilícitas durante a vida segundo o gênero.

\begin{tabular}{|c|c|c|c|c|c|}
\hline \multirow[t]{3}{*}{ Condutas } & \multicolumn{4}{|c|}{ Sexo } & \multirow[t]{3}{*}{ Valor de p } \\
\hline & \multicolumn{2}{|c|}{ Masculino } & \multicolumn{2}{|c|}{ Feminino } & \\
\hline & n (116) & $\%$ & n (266) & $\%$ & \\
\hline Consumo de qualquer forma de cocaína, incluindo pó, crack ou freebase & 2 & 1,7 & 4 & 1,5 & 1,000 * \\
\hline $\begin{array}{l}\text { Consumo de inalante aerossol ou qualquer tinta ou spray } \\
\text { para ficar fora da realidade }\end{array}$ & 43 & 37,1 & 41 & 15,4 & $<0,001 * *, * * *$ \\
\hline Uso de pílulas ou injeção de esteróides sem uma prescrição médica \# & 15 & 13,0 & 12 & 4,5 & $0,003 * \star * * \star *$ \\
\hline $\begin{array}{l}\text { Uso de qualquer tipo de droga ilícita como LSD, PCP, ecstasy, cogumelo, } \\
\text { anfetamina (speed), anfetamina (ice) e ou heroína }\end{array}$ & 8 & 6,9 & 6 & 2,3 & 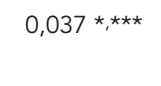 \\
\hline Consumo maconha (uso na vida) & 30 & 25,9 & 35 & 13,2 & $0,002 * \star \star * \star \star *$ \\
\hline Consumo de maconha nos últimos 30 dias \#\# & 7 & 23,3 & 8 & 22,9 & $0,964 * \star$ \\
\hline
\end{tabular}

* Emprego do teste exato de Fisher;

** Emprego do teste qui-quadrado de Pearson;

$\star \star \star$ Associação significante a $5 \%$;

\# Dois pesquisados não responderam à questão (1 masculino, 1 feminino);

\#\# 317 pesquisados nunca fumaram maconha durante a vida (86 masculinos e 231 femininos)

\section{Discussão}

De uma maneira geral, as mulheres apresentaram freqüências mais baixas de condutas de risco como: consumo de álcool, uso do tabaco e de drogas ilícitas, além de porte de arma e envolvimento em briga física.

Em outros estudos também se observou maior prevalência de tabagismo entre jovens do gênero masculino de outros países, estudan- tes de cursos de saúde 6,7 e maior consumo de drogas ilícitas entre jovens de outros estados do Brasil 10,14.

As condutas relacionadas à segurança no trânsito, suicídio e hábitos alimentares se mostraram semelhantes entre os jovens, independente do gênero. Em relação à segurança no trânsito, o resultado mostra discordância do estudo de Andrade et al. 5 com estudantes de um curso de medicina na Região Sul do Brasil, no qual 
Avaliação do comportamento sexual segundo o gênero.

\begin{tabular}{|c|c|c|c|c|c|}
\hline \multirow[t]{3}{*}{ Comportamento sexual } & \multicolumn{4}{|c|}{ Gênero } & \multirow[t]{3}{*}{ Valor de $\mathrm{p}$} \\
\hline & \multicolumn{2}{|c|}{ Masculino } & \multicolumn{2}{|c|}{ Feminino } & \\
\hline & n (116) & $\%$ & n (266) & $\%$ & \\
\hline Experiência na vida * & 112 & 96,6 & 210 & 79,8 & $<0,001 \star \star$ \\
\hline \multicolumn{6}{|l|}{ 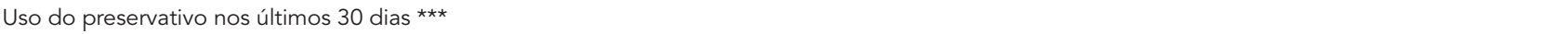 } \\
\hline Não teve relação sexual nos últimos 30 dias & 15 & 13,5 & 32 & 15,4 & 0,068 ** \\
\hline Nunca & 16 & 14,4 & 34 & 16,3 & \\
\hline Raramente/Às vezes & 28 & 25,2 & 75 & 36,1 & \\
\hline A maioria das vezes/Sempre & 52 & 46,8 & 67 & 32,2 & \\
\hline \multicolumn{6}{|c|}{ Método contraceptivo usado na última relação sexual \# } \\
\hline Nenhum método foi usado & 11 & 9,8 & 14 & 6,7 & 0,320 ** \\
\hline Pílulas anticoncepcionais & 43 & 38,4 & 118 & 56,5 & $0,002 * \star$ \\
\hline Preservativo & 53 & 47,3 & 80 & 38,3 & 0,117 ** \\
\hline Coito interrompido & 11 & 9,8 & 35 & 16,7 & $0,091 \star \star$ \\
\hline Algum outro método & 8 & 7,1 & 10 & 4,8 & 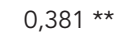 \\
\hline Não sabe & 2 & 1,8 & 1 & 0,5 & $0,280 \# \#$ \\
\hline Já ter feito o teste HIV \#\#\# & 52 & 44,8 & 70 & 26,5 & $0,001 \# \#$ \\
\hline
\end{tabular}

* Três pesquisados (femininos) não responderam à questão;

** Emprego do teste qui-quadrado de Pearson;

*** 63 pesquisados não responderam à questão ( 5 masculinos e 58 masculinos);

\# 61 pesquisados não responderam à questão (4 masculinos e 57 femininos);

\#\# Emprego do teste exato de Fisher;

\#\#\# Dois pesquisados (femininos) não responderam à questão.

Tabela 5

Avaliação da alimentação, atividade física e do peso segundo o gênero.

\begin{tabular}{|c|c|c|c|c|c|}
\hline \multirow[t]{3}{*}{ Condutas } & \multicolumn{4}{|c|}{ Gênero } & \multirow[t]{3}{*}{ Valor de $p$} \\
\hline & \multicolumn{2}{|c|}{ Masculino } & \multicolumn{2}{|c|}{ Feminino } & \\
\hline & n (116) & $\%$ & n (266) & $\%$ & \\
\hline Consumo de frutas ou suco de frutas & 88 & 75,9 & 202 & 75,9 & 0,987 * \\
\hline Consumo de salada de verdura/vegetais cozidos & 66 & 56,9 & 129 & 48,5 & 0,131 * \\
\hline $\begin{array}{l}\text { Consumo de hambúrguer, coxinha, empadinhas, } \\
\text { cachorro-quente ou salgadinhos }\end{array}$ & 59 & 50,9 & 121 & 45,5 & $0,333 *$ \\
\hline Consumo de biscoitos, tortas, bolos ou doces & 89 & 76,7 & 209 & 78,6 & 0,689 * \\
\hline Prática de exercícios ou esportes & 70 & 60,3 & 94 & 35,3 & $<0,001$ * \\
\hline \multicolumn{6}{|l|}{ Auto-avaliação do peso } \\
\hline Abaixo do peso normal & 18 & 15,5 & 44 & 16,5 & $0,885 *$ \\
\hline No peso certo & 54 & 46,6 & 128 & 48,1 & \\
\hline Acima do peso normal & 44 & 37,9 & 94 & 35,3 & \\
\hline \multicolumn{6}{|l|}{ Atitude em relação a seu peso } \\
\hline Perder peso & 29 & 25,0 & 113 & 42,5 & $<0,001$ * \\
\hline Ganhar peso & 31 & 26,7 & 22 & 8,3 & \\
\hline Manter peso & 29 & 25,0 & 84 & 31,6 & \\
\hline Não está tentando fazer nada & 27 & 23,3 & 47 & 17,7 & \\
\hline
\end{tabular}

* Emprego do teste qui-quadrado de Pearson. 
os homens relataram dirigir e consumir álcool mais freqüentemente. No estudo de Näslund \& Fredrikson 15, as mulheres suecas também apresentaram condutas mais positivas com relação à segurança no trânsito, como: uso do cinto de segurança mais habitualmente, respeito aos limites de velocidade e um menor percentual de mulheres relataram dirigir após o consumo de bebidas alcoólicas.

Com relação ao consumo de bebida alcoólica, verificou-se que, apesar de os homens terem relatado consumo mais elevado na vida, não se verificou diferença significativa quando se considerou o período recente, ou seja, os trinta dias que antecederam a pesquisa. Resultado semelhante ocorreu com relação ao consumo de maconha. Este resultado não coincidiu com os estudos de Arliss ${ }^{8}$ com jovens americanos nem com os de Lemos et al. ${ }^{16}$ com estudantes de Salvador, Bahia, nos quais os homens apresentaram um maior consumo recente de bebida alcoólica.

O tabagismo foi mais prevalente entre os homens, em concordância com o estudo de Takeda et al. 7; a tentativa de parar de fumar foi, no entanto, mais prevalente entre as mulheres. Resultado semelhante foi encontrado no estudo de Vakeflliu et al. 6 , pois, apesar de a maioria dos estudantes de medicina terem demonstrado conhecimento de que o tabagismo é perigoso para a saúde, quase metade dos estudantes no final do curso eram fumantes, especialmente aqueles do gênero masculino, coincidindo com os dados coletados neste estudo.

Os homens também consumiram significativamente com mais freqüência drogas ilícitas do que as mulheres, em concordância com outros estudos realizados no Brasil 10,14,16.

Referentemente ao comportamento sexual, embora a maioria dos estudantes tenham vida sexual ativa, menos da metade dos estudantes do gênero masculino relataram o uso do preservativo "na maioria das vezes" ou "sempre", o que concorda com os resultados encontrados por Palma et al. 17 entre universitários de saúde no Rio de Janeiro.

Os estudantes do gênero masculino apresentaram condutas de maior risco para saúde em alguns temas que segundo Mahalik et al. 18 se deve ao encorajamento da sociedade machista ocidental de o homem adotar tais comportamentos. Estes autores ainda sugerem a realização de estudos qualitativos que esclareçam estas diferenças entre os gêneros.

Ainda que a maioria dos estudantes tenha relatado ter recebido informação durante o curso de graduação sobre prevenção de doenças sexualmente transmissíveis, as condutas relacionadas à prática sexual mostraram-se, no presente estudo, inseguras. Dessa maneira, fica evidente que a abordagem deste tema não foi eficiente, sugerindo que as estratégias devam ser modificadas para que possam alcançar um melhor resultado em relação ao comportamento sexual seguro dos próprios estudantes.

Apesar de não se ter observado diferença significativa entre os gêneros quanto à autoavaliação do peso, as mulheres relataram maior preocupação em perder ou manter o peso. Esta maior preocupação com o peso corporal pelas mulheres também foi relatada por Herman et al. 9. Todavia a prática de atividade física foi significativamente mais freqüente entre os homens $1,3,7,11,17,19$

Independentemente do gênero, deve-se considerar, ainda, que os pesquisados eram universitários de cursos da área de saúde, que deverão em suas atividades profissionais orientar e aconselhar para a adoção de hábitos saudáveis. Questiona-se o papel da universidade neste contexto, tanto quanto as implicações das condutas de saúde dos estudantes em sua prática futura.

\section{Considerações finais}

Pode-se concluir que homens e mulheres apresentam condutas de saúde diferentes, sugerindo a necessidade de uma abordagem diferenciada e a elaboração de estratégias de promoção de saúde adequadas para cada gênero. Recomenda-se a realização de estudos qualitativos que investiguem os fatores associados às diferenças entre gêneros entre jovens universitários. 


\section{Resumo}

Este estudo investigou diferenças entre os gêneros nas condutas de saúde de universitários em final de curso. A amostra foi composta de 382 estudantes de universidades públicas do Estado de Pernambuco, Brasil, com idade entre 20 e 29 anos. Os dados foram coletados mediante a aplicação do questionário National College Health Risk Behavior Survey, validado previamente para o português. Foram utilizadas técnicas de estatística descritiva e inferencial. Para análise de associação foram utilizados teste qui-quadrado ou exato de Fisher. Os resultados foram considerados significantes para $p<0,05$. Verificou-se que, de modo geral, os estudantes do gênero feminino apresentaram freqüências menores de condutas de risco para a saúde como: consumo de álcool ( $p=0,005)$, tabagismo $(p=0,002)$, experimentação de maconha $(p=0,002)$, consumo de inalantes $(p<0,001)$, uso de esteróides ( $p=0,003)$, porte de arma $(p=0,001)$ e envolvimento em briga física $(p=0,014)$. Os estudantes, no entanto, relataram maior preocupação em perder ou manter o peso, apesar de a prática de atividade física ter sido menos freqüente neste grupo. Pode-se concluir que há diferenças significativas com relação às condutas de saúde entre os gêneros, devendo haver abordagens diferenciadas para os dois grupos.

Estudantes de Ciências da Saúde; Conduta de Saúde; Fatores de Risco; Gênero

\section{Referências}

1. Lee RLT, Loke AJTY. Health-promoting behaviors and psychosocial well-being of university students in Hong Kong. Public Health Nurs 2005; 22:209-20.

2. Rugkasa J, Stewart-Knox B, Sittlington J, Abaunza PS, Treacy MP. Hard boys, attractive girls: expressions of gender in young people's conversations on smoking in Northern Ireland. Health Promot Int 2003; 18:307-14.

3. Lowry R, Galuska DA, Fulton JE, Wechsler H, Kann L, Collins JL. Physical activity, food choice, and weight management goals and practices among U.S. college students. Am J Prev Med 2000; 18: 18-27.

4. Davies J, McCrae BP, Frank J, Dochnahl A, Pickering $\mathrm{T}$, Harrison B, et al. Identifying male college students' perceived health needs, barriers to seeking help, and recommendations to help men adopt healthier lifestyles. J Am Coll Health 2000; 48:259-67.

5. Andrade SM, Soares DA, Braga GP, Moreira JH, Botelho FMN. Comportamentos de risco para acidentes de trânsito: um inquérito entre estudantes de medicina na Região Sul do Brasil. Rev Assoc Méd Bras 2003; 49:439-44.

\section{Colaboradores}

V. Colares participou da elaboração do projeto de pesquisa, análise de dados e revisão final do texto. C. Franca participou da elaboração do projeto de pesquisa, coleta e análise dos dados e redação do texto. E. Gonzalez participou da coleta de dados e redação do texto.
6. Vakeflliu Y, Argjiri D, Peposhi I, Agron S, Melani AS. Tobacco smoking habits, beliefs, and attitudes among medical students in Tirana, Albania. Prev Med 2002; 34:370-3.

7. Takeda Y, Kawachi I, Yamagata Z, Hashimoto S, Matsumura Y, Oguri S, et al. The impact of multiple role occupancy on health-related behaviours in Japan: differences by gender and age. Public Health 2006; 120:966-75.

8. Airliss RM. Cigarette smoking, binge drinking, physical activity, and diet in 138 Asian American and Pacific Islander community college students in Brooklyn, New York. J Community Health 2007; 32:71-84.

9. Hermàn $\mathrm{M}$, Fernàndez $\mathrm{A}$, Ramos $\mathrm{M}$. La salud de los jóvenes. Parte I. La salud y el sistema sanitario desde la perspective de gênero y classe social. Gac Sanit 2004; 18 Suppl:47-55.

10. Silva LVER, Malbergier A, Stempliuk VA, Andrade AG. Fatores associados ao consumo de álcool e drogas entre estudantes universitários. Rev Saúde Pública 2006; 40:280-8. 
11. Dunn M, Wang MQ. Effects of physical activity on substance use among college students. American Journal of Health Studies 2003; 18:126-32.

12. Pirotta KCM, Schor N. Intenções reprodutivas e práticas de regulação da fecundidade entre universitários. Rev Saúde Pública 2004; 38:495-502.

13. Centers for Disease Control and Prevention. Healthy youth! http://www.cdc.gov/healthyyouth/ about/mission.htm (acessado em 15/Jul/2005).

14. Lucas ACS, Parente RCP, Picanço NS, Conceição DA, Costa KRC, Magalhães IRS, et al. Uso de psicotrópicos entre universitários da área da saúde da Universidade Federal do Amazonas, Brasil. Cad Saúde Pública 2006; 22:663-71.

15. Näslund GK, Fredrikson M. Health behavior, knowledge and attitudes among Swedish university students. Scand J Psychol 1993; 34:197-211.
16. Lemos KM, Neves NMBC, Kuwano AY, Tedesqui G, Bitencourt GV, Neves FBCS, et. al. Uso de substâncias psicoativas entre estudantes de Medicina de Salvador (BA). Rev Psiquatr Clín (São Paulo) 2007; 34:118-24.

17. Palma A, Abreu RA, Cunha CA. Comportamento de risco e vulnerabilidade entre estudantes de educação física. Rev Bras Epidemiol 2007; 10:117-26.

18. Mahalik JR, Burns SM, Syzdek M. Masculinity and perceived normative health behaviors as predictors of men's health behaviors. Soc Sci Med 2007; 64:2201-9.

19. Sales-Costa R, Heilborn ML, Werneck GL, Faerstein E, Lopes CS. Gênero e prática de atividade física de lazer. Cad Saúde Pública 2003; 19 Suppl 2:S325-33.

Recebido em 10/Jun/2008

Versão final reapresentada em 05/Set/2008 Aprovado em 01/Out/2008 